\title{
Possible ionospheric preconditioning by shear flow leading to equatorial spread $F$
}

\author{
D. L. Hysell ${ }^{1}$, E. Kudeki ${ }^{2}$, and J. L. Chau ${ }^{3}$ \\ ${ }^{1}$ Department of Earth and Atmospheric Sciences, Cornell University, Ithaca, NY 14853, USA \\ ${ }^{2}$ Department of Electrical and Computer Engineering, University of Illinois, Urbana, Il 61801, USA \\ ${ }^{3}$ Radio Observatorio de Jicamarca, Instituto Geofísico del Perú, Lima, Perú
}

Received: 18 April 2005 - Revised: 28 July 2005 - Accepted: 3 August 2005 - Published: 14 October 2005

\begin{abstract}
Vertical shear in the zonal plasma drift speed is apparent in incoherent and coherent scatter radar observations of the bottomside $F$ region ionosphere made at Jicamarca from about 1600-2200 LT. The relative importance of the factors controlling the shear, which include competition between the $E$ and $F$ region dynamos as well as vertical currents driven in the $E$ and $F$ regions at the dip equator, is presently unknown. Bottom-type scattering layers arise in strata where the neutral and plasma drifts differ widely, and periodic structuring of irregularities within the layers is telltale of intermediate-scale waves in the bottomside. These precursor waves appear to be able to seed ionospheric interchange instabilities and initiate full-blown equatorial spread $F$. The seed or precursor waves may be generated by a collisional shear instability. However, assessing the viability of shear instability requires measurements of the same parameters needed to understand shear flow quantitatively - thermospheric neutral wind and off-equatorial conductivity profiles.
\end{abstract}

Keywords. Ionosphere (Equatorial ionosphere; ionospheric irregularities) - Space plasma physics (Waves and instabilities)

\section{Introduction}

A new perspective on the stability of the postsunset equatorial $F$ region ionosphere is emerging from experiments conducted at the Jicamarca Radio Observatory near Lima, Peru. The Jicamarca incoherent scatter radar has specialized capabilities for observing both the ionospheric conditions that lead to equatorial spread $F$ and for monitoring the plasma irregularities appearing subsequently. Being able to observe incoherent scatter looking perpendicular to the geomagnetic field, the $50 \mathrm{MHz}$ radar can exploit the long correlation time of the signal to make very accurate measurements of the line- of-sight plasma drift speed (Woodman, 1970). Using a dual beam configuration, unambiguous profiles of the plasma vector drift velocity in the plane perpendicular to $\mathbf{B}$ can also be obtained. The modularity of the Jicamarca phased array antenna permits the application of aperture synthesis imaging, which yields unambiguous two- or three-dimensional images of the spatial configuration of plasma irregularities producing field-aligned backscatter (Kudeki and Sürücü, 1991; Woodman, 1997).

A striking characteristic of the bottomside $F$ region ionosphere extending from late afternoon to a few hours before midnight is shear flow, as first noted by Kudeki et al. (1981) and Tsunoda et al. (1981). Plasma drifts westward in the bottomside $F$ region while drifting eastward near and above the $F$ peak, with the shear being strongest just after sunset. Shear flow was studied theoretically by Haerendel et al. (1992) and Haerendel and Eccles (1992) and is a component of the postsunset vortex described recently by Kudeki and Bhattacharyya (1999) and Eccles et al. (1999) using radar and satellite observations, respectively. Constraints imposed by low latitude current closure in the vicinity of the solar terminator ultimately control shear flow, but the phenomenon is not well accounted for quantitatively.

In this manuscript, we review recent findings suggesting that shear flow may destabilize the equatorial $F$ region, possibly preconditioning it for interchange instabilities responsible for spread $F$ (as postulated by Maruyama et al., 2002). If so, then monitoring plasma flows and anticipating shear instability may provide a means of forecasting some instances of spread $F$. Since the thermospheric neutral wind speed both drives the plasma flow and influences shear instability, monitoring neutral circulation will be necessary. The C/NOFS satellite therefore has a crucial role to play in this analysis. However, additional ground-based instrumentation will be required for measuring off-equatorial conductivity, which both influences shear flow and inhibits instability. 


\section{Examples of shear flow}

Figure 1 shows examples of ionospheric structure and circulation from paired events observed in November or December 2001, 2002, and 2003. The data were acquired using refined experimental techniques introduced recently by Kudeki et al. (1999). The top panel shown for each event represents relative backscatter power in grayscale logarithmic format. Although the backscatter power has been scaled by the square of the range, it is not calibrated, is distorted to some degree by magnetoionic effects, and is meant to serve here only as a rough estimate of the relative electron density in undisturbed regions of the ionosphere. Regions of intense backscatter signify coherent scatter from plasma irregularities and are plotted using different amplitude scaling.

The middle panel of each event in Fig. 1 shows vertical velocity derived from the average Doppler shift of Jicamarca's dual beams, which are directed approximately east and west of magnetic zenith for this experiment. The bottom panel shows zonal velocity derived from the difference of the dual beam Doppler shifts. Both coherent and incoherent scatter data were utilized in generating the panels. All the data were derived from geomagnetically quiet periods.

The three examples on the left side of Fig. 1 represent events when ionospheric irregularities were confined to narrow layers in the bottomside. These "bottom-type" layers are nearly ubiquitous immediately after sunset during equinox and December solstice in the Peruvian sector but do not represent significant ionospheric disruptions and are not associated with strong radio scintillations. In contrast, the three examples on the right portray occurrences of "full-blown" equatorial spread $F$. Forecasting topside spread $F$ events like these on the basis of available data is a goal of the space weather community. Forecast strategies have often involved applying a threshold condition to the intensity of the prereversal enhancement of the vertical drift. Ascent drives the $F$ layer to altitudes with reduced ion-neutral collision frequencies more susceptible to gravitational interchange instability. Cursory examination of the data here alone suggest that such a strategy should have merit but would be fallible and could not be applied before about 23:30 UT (18:30 LT) or only about an hour before the onset of instability. Discriminating between plasma ascent leading to instability and ascent caused by instability would also be problematic.

Shear flow is evident in all of the events in Fig. 1 and is most obvious after about 00:30 UT (19:30 LT) when backscatter from bottom-type layers points to rapid westward motion immediately below regions of rapid eastward motion. It might seem fortuitous that the bottom-type layers exist to provide a radar target, since the valley region plasma is insufficiently dense to be monitored using incoherent scatter. However, Kudeki and Bhattacharyya (1999) pointed out that, assuming the thermospheric winds are eastward throughout the $F$ region, the bottom-type layers occupy strata where the plasma drifts are strongly retrograde. Given that significant horizontal density gradients are also present in these strata around sunset due to photochemical and dynamical effects, the strata would be prone to horizontal wind-driven interchange instabilities. They hypothesized that such instabilities are responsible for the bottom-type scattering layers, which are byproducts of retrograde drifts in the bottomside and therefore not merely fortuitous.

The Kudeki and Bhattacharyya (1999) theory explains why the bottom-type layers exhibit no vertical development, as exemplified by the RTI map in Fig. 2. Since the primary plasma waves grow by advection, they always remain confined to the strata where the drifts are retrograde. In contrast, interchange instabilities driven by gravity or a zonal electric field must grow by convection, as did the two radar plumes passing over Jicamarca later in the same event. Another universal characteristic of the layers is that they vanish at the first appearance of topside spread $F$ irregularities and seldom reappear. This feature is at least partially explained by theory, since retrograde plasma drifts arise ultimately from the demands of the current system near the solar terminator and should cease by some local time. Topside plumes likely disrupt the local circulation and break up any bottom-type layers nearby.

Further evidence that wind-driven instability is at work in the bottomside was provided by Hysell et al. (2004), who used aperture synthesis imaging techniques to analyze the structure of the primary waves in bottom-type scattering layers. They found that the primary waves were kilometric in scale and generally horizontally oriented and elongated rather than vertically. The primary waves they observed appeared to grow by advection rather than convection, consistent with the wind driven interchange instability theory.

Moreover, Hysell et al. (2004) found that the primary plasma waves in bottom-type layers sometimes cluster together in regular, wavelike patches separated horizontally by about $30 \mathrm{~km}$. Clustering took place only in those events when topside spread $F$ occurred later. They associated the clustering with a horizontal wave in the background plasma density that would present alternately stable and unstable horizontal gradients for wind-driven interchange instability. Figure 3 shows a radar image derived from the bottom-type layer in Fig. 2 illustrating such clustering.

Finally, Hysell et al. (2004) concluded that the clustered or patchy bottom-type layers were telltale of decakilometric waves in the bottomside $F$ region that could serve to precondition or seed the ionosphere for gravitational interchange instabilities (Rayleigh Taylor) to follow. This hypothesis was supported by the fact that the intermediate-scale plasma irregularities that formed later in the spread $F$ events shared the same decakilometric wavelength. This suggests a forecast strategy for spread $F$ based on detecting clustering in bottom-type layers immediately after sunset. The strategy is being evaluated for its merits but in no event would be applicable before about 19:30 LT when the layers first appear.

The $\sim 30 \mathrm{~km}$ wavelength of the undulations in the bottomside $F$ region inferred from the patchy bottom-type scatterers does not correspond to a characteristic scale length of the ionospheric interchange instability. This is why it is seen as preconditioning rather than just the early stages of instability. 

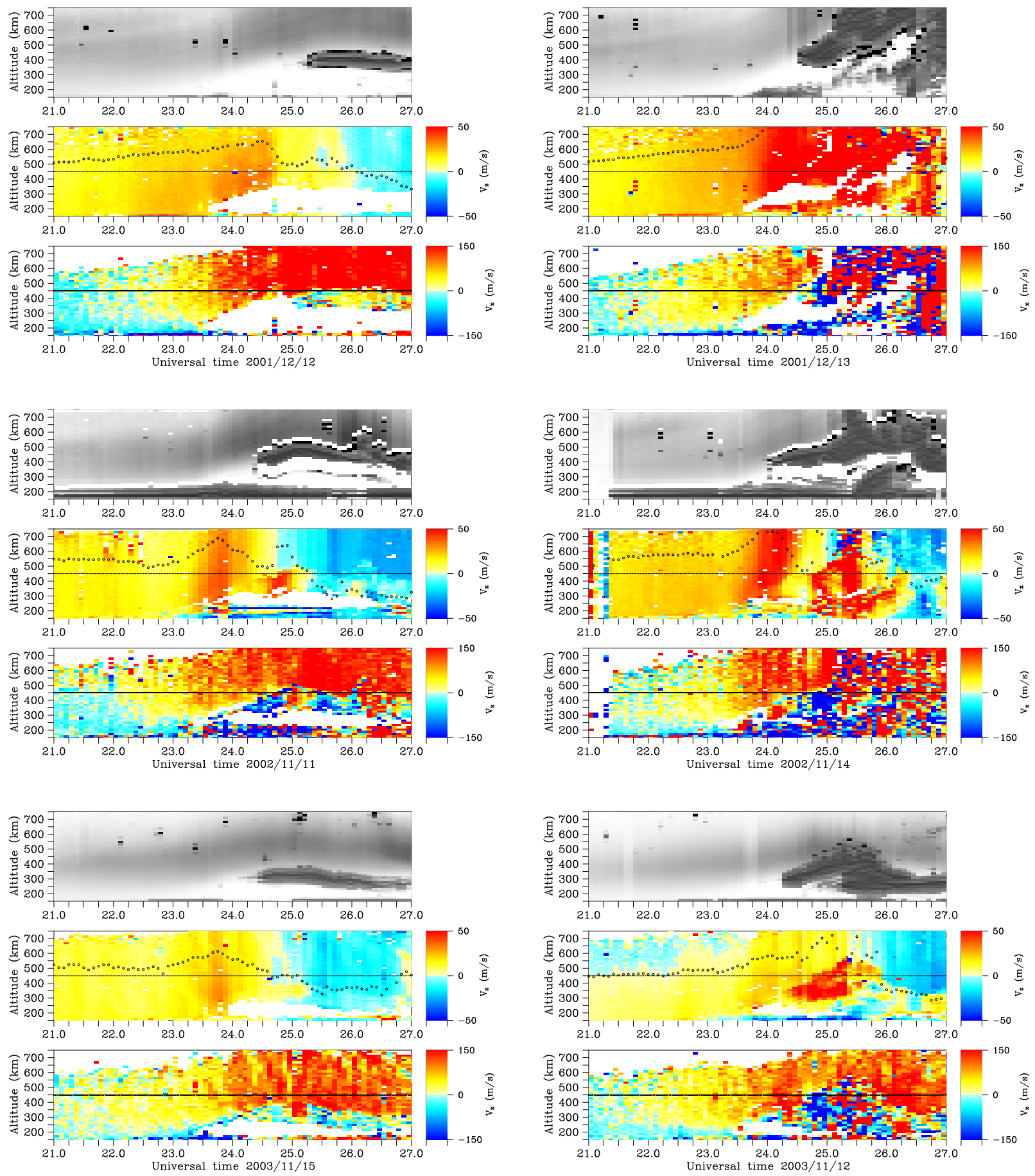

Fig. 1. Six examples of ionospheric conditions around twilight. Each example shows the backscatter power (top panel), vertical plasma drift (middle panel), and zonal plasma drift (lower panel), plotted against the scales shown. Symbols plotted in the middle panel represent the vertical drift velocity at $450 \mathrm{~km}$ altitude for reference. The left and right columns portray events when topside spread $F$ did not and did occur, respectively. Note that $\mathrm{UT}=\mathrm{LT}+5 \mathrm{~h}$.

Preconditioning or seeding of the postsunset ionosphere is often attributed to gravity waves (Kelley et al., 1981). This hypothesis is difficult to test experimentally, however, since only the effects of gravity waves and not the gravity waves themselves can be detected in the thermosphere using ISRs.
Recently, Vadas and Fritts (2004) examined the issue theoretically, showing that a spectrum of gravity waves launched by mesoscale convection cells could survive wind shears and viscous and conductive damping and penetrate into the lower thermosphere. However, the $30 \mathrm{~km}$ wavelength is at the 


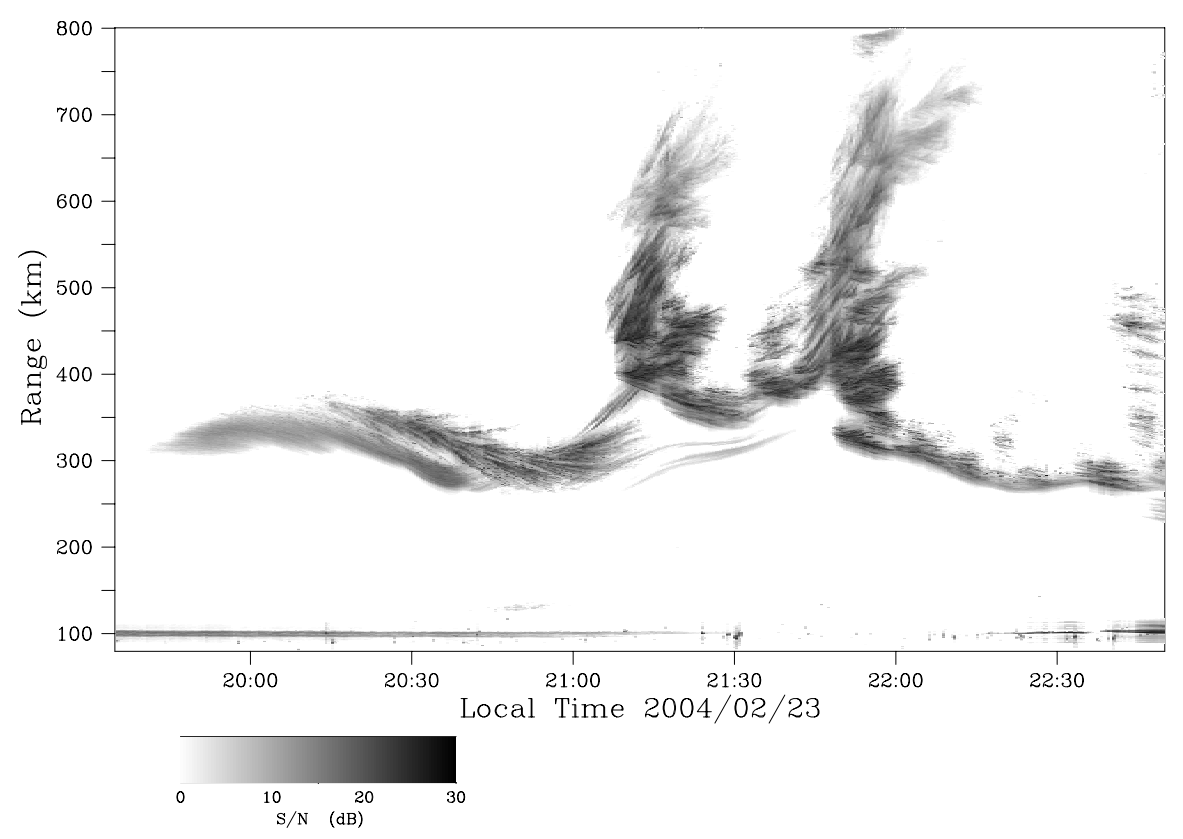

Fig. 2. Range time intensity (RTI) image of a topside spread $F$ event. Only a bottom-type layer was present prior to about 21:00 LT.

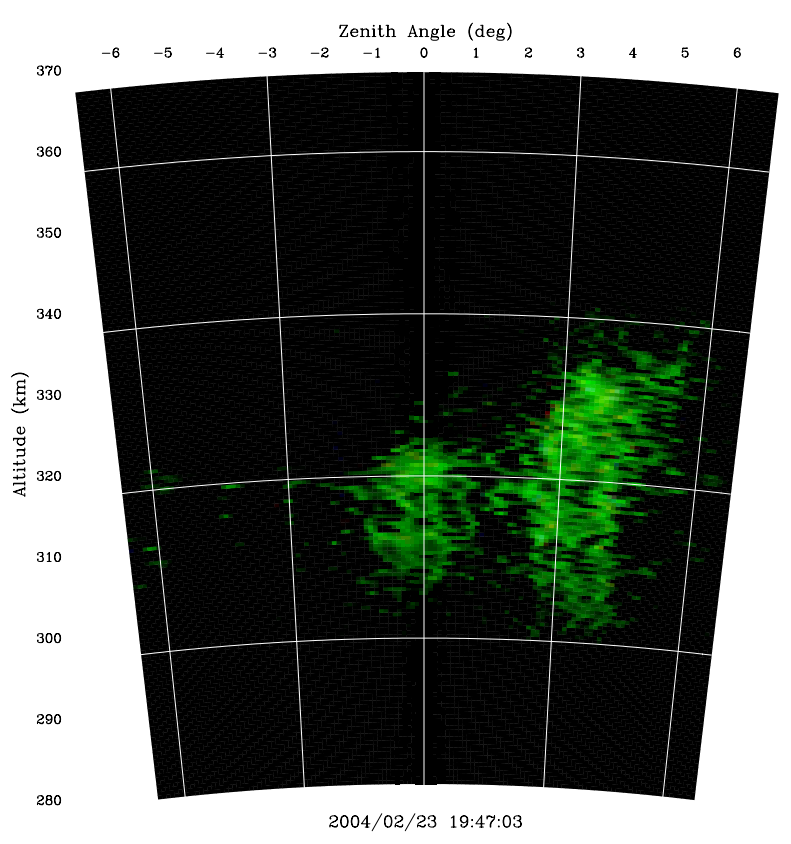

Fig. 3. Radar image of plasma irregularities within the bottom-type layer seen in the previous figure. The irregularities are shown at the moment they first drifted westward into the radar illuminated volume.

extreme short wavelength edge of allowed wavelengths, and nothing in their theory would tend to favor it.

Meanwhile, the $30 \mathrm{~km}$ wavelength is roughly comparable to the vertical scale length of the plasma shear flow. Recently, Hysell and Kudeki (2004) examined the possibility that shear flow may cause the bottomside $F$ region to become unstable and produce the precursor waves inferred from radar imag- ing. If so, then shear flow itself could precondition the ionosphere for full-blown spread $F$. A quantitative understanding of the shear instability might therefore lead to a forecast strategy for spread $F$ with the potential for advanced warning, given that shear develops early in the evening. Below, we review the causes of shear flow in the equatorial ionosphere, formulate the shear instability problem, and compose an approximate but closed-form expression for the instability growth rate.

\section{Origins of shear flow}

That shear flow should exist in the bottomside equatorial $F$ region was evident in the numerical modeling results of Heelis et al. (1974), argued on theoretical grounds by Fejer (1981), and observed by Kudeki et al. (1981) and Tsunoda et al. (1981). This shear can be understood, at least partly, in terms of dynamo theory.

A model of the ionospheric dynamo was described by Richmond (1973) who assumed a dipole, equipotential model for the geomagnetic field and considered an individual flux tube. Quasineutrality in a plasma imposes the constraint that the total current density be solenoidal $(\nabla \cdot \mathbf{J}=0)$, where

$$
\begin{aligned}
\mathbf{J} & =\sigma_{P}\left(\mathbf{E}_{\perp}+\mathbf{u} \times \mathbf{B}\right) \\
& +\sigma_{H} \hat{b} \times\left(\mathbf{E}_{\perp}+\mathbf{u} \times \mathbf{B}\right)+\sigma_{\circ} \mathbf{E}_{\|}
\end{aligned}
$$

is the current density and $\sigma_{P}, \sigma_{H}$, and $\sigma_{\circ}$ are the Pedersen, Hall, and direct conductivities, respectively. Also, $\mathbf{E}$ is the electric field, $\mathbf{u}$ is the neutral wind velocity, and $\hat{b}$ is a unit vector parallel to the geomagnetic field $\mathbf{B}$.

Application of the quasineutrality condition to the current density within a magnetic flux tube, neglect of horizontal 
variations in the fields and conductivities, and integration of the result over the coordinate parallel to $\mathbf{B}$ while taking the magnetic field lines to be equipotentials leads directly to the following equation governing the vertical ionospheric electric field:

$$
\begin{aligned}
E_{p} & =\left(E_{\phi} h_{\phi} \int \sigma_{H} h_{q} d q\right. \\
& -\int\left(\sigma_{P} u_{\phi}+\sigma_{H} u_{p}\right) B h_{\phi} h_{q} d q \\
& \left.+\frac{d I}{d \phi}\right) /\left(h_{p} \int \sigma_{P} \frac{h_{\phi} h_{q}}{h_{p}} d q\right)
\end{aligned}
$$

written here using the orthogonal coordinate system $(p, q, \phi)$, where $p$ is normal to the dipole magnetic field and in the plane containing the dipole moment, $q$ is parallel to the field line, and $\phi$ is the magnetic longitude. The $h$ factors are the coordinate scale factors (see for example Hysell et al. (2004). Also, $d I / d \phi$ represents the differential current density passing through the flux tube in the $p$ direction. The current pattern involved in producing $E_{p}$ is informatively sketched in Fig. 9 of Haerendel and Eccles (1992).

Equation (2) succinctly summarized the factors that influence the vertical electric field in the equatorial ionosphere. These factors have been discussed individually in theoretical and experimental contexts by a number of investigators including Anandarao et al. (1978); Fejer (1981); Stenning (1981); Takeda and Maeda (1983); Farley et al. (1986) and by Haerendel et al. (1992), Haerendel and Eccles (1992), and recently by Eccles (1998) and Eccles et al. (1999) in more complete theoretical treatments. They include 1) zonal electric fields on flux tubes with significant Hall conductivity, as are responsible for driving the equatorial electrojet, 2) zonal winds on flux tubes with significant Pedersen conductivity, as drive the $E$ and $F$ region dynamos, 3) vertical winds, a largely unknown quantity, and 4) vertical boundary currents forced from above or below the flux tube in question. In the bottomside and valley regions around twilight, this last factor could result from the closure of the equatorial electrojet, which must turn partly vertical at the boundary of the evening terminator. It could also result from the $F$ region dynamo operating in flux tubes near the $F$ peak. The finite efficiency of the dynamo implies the existence of an upward vertical current there. The demands imposed by this current can only be supported at lower altitudes, where the conductivity is smaller, by a potentially large vertical electric field (Haerendel et al., 1992; Haerendel and Eccles, 1992).

Which of these effects dominates in the postsunset bottomside? Haerendel et al. (1992) attribute most of the shear there to the boundary current effect. They also considered the effect of zonal electric fields, which generate vertical polarization electric fields on flux tubes with significant integrated Hall conductivities in accordance with Eq. (2). This mechanism was deemed to be effective mainly in the trough region of the $F_{1}$ layer and below. While Haerendel et al. (1992) did not discount the importance of the $E$ region dynamo in producing shears and westward $F$ region drifts altogether, they restricted the domain of importance to the valley region. However, this appraisal was based on $E$ region neutral wind estimates of only $25 \mathrm{~m} / \mathrm{s}$ which we now recognize to be a drastic underestimate, by a factor of five or more, in light of recent chemical release experiments (Larsen and Odom, 1997).

To summarize, although the various factors that can influence shear flow in the ionosphere are individually understood, their relative importance is not. Resolution of the problem evidently requires specification of both the neutral wind profile in the mesosphere, lower thermosphere, and thermosphere as well as the off-equatorial conductivity profiles.

\section{Shear-induced instability}

Hysell and Kudeki (2004) recently addressed the stability of the $F$ region ionosphere in a sheared flow with an eigenvalue analysis. Keskinen et al. (1988) had already shown that electrostatic Kelvin Helmholtz instabilities are stabilized by ionneutral collisions in the auroral ionosphere, but that analysis did not explore the collisional branch of the instability in the limit of steep plasma density gradients as are found in the bottomside equatorial $F$ region. Following Keskinen et al. (1988), Hysell and Kudeki (2004) neglected Hall and parallel currents but allowed for polarization currents in their two-dimensional analysis:

$\mathbf{J}=\frac{n e}{\Omega_{i} B}\left[v_{\text {in }}(\mathbf{E}+\mathbf{u} \times \mathbf{B})+\left(\frac{\partial}{\partial t}+\mathbf{v} \cdot \nabla\right) \mathbf{E}\right]$

The quasineutrality condition, together with the ion continuity equation, formed the basis of their model. Linearization of the model was performed according to

$n(x, z)=n_{\circ}(z)+n_{1}(z) e^{i\left(k_{x} x-\omega t\right)}$
$\phi(x, z)=\phi_{\circ}(z)+\phi_{1}(z) e^{i\left(k_{x} x-\omega t\right)}$

where terms with subscripts 0 and 1 represent zero- and firstorder quantities, respectively, and where the Cartesian coordinates $x, y$, and $z$ represented the eastward, northward, and upward directions at the magnetic equator. The linearized model equations took the form:

$$
\begin{aligned}
& \left(\omega-k_{x} v_{\circ}\right) n_{1}=-\frac{k_{x}}{B} \frac{d n_{\circ}}{d z} \phi_{1} \\
& \left(\omega+i v_{\text {in }}-k_{x} v_{\circ}\right)\left[\frac{d}{d z}\left(n_{\circ} \frac{d \phi_{1}}{d z}\right)-n_{\circ} k_{x}^{2} \phi_{1}\right] \\
& =-i n_{\circ} \frac{d v_{\text {in }}}{d z} \frac{d \phi_{1}}{d z}-k_{x} \frac{d}{d z}\left(n_{\circ} \frac{d v_{\circ}}{d z}\right) \phi_{1} \\
& +i \frac{d}{d z}\left(B v_{\text {in }}\left(u-v_{\circ}\right) n_{1}\right)
\end{aligned}
$$

where $\omega$ is the complex frequency and serves as the eigenvalue, $k_{x}$ is the assumed horizontal wavenumber, $v_{\circ}$ is the background horizontal plasma flow speed arising from gradients in the zero-order electrostatic potential, and $v_{\text {in }}$ is the ion-neutral collision frequency. 


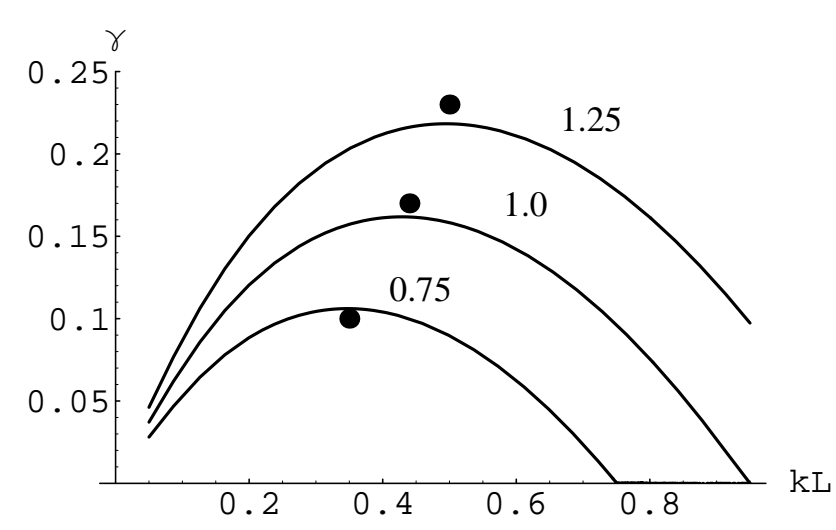

Fig. 4. Normalized growth rate $\gamma$ (scaled to $V_{\circ} / L$ ) versus normalized wavenumber $k L$ given $u=2$ and $\nu_{\circ}=2$ for three different values of $v_{1}$. The points represent (9) evaluated at the value of $k_{x}$ indicated and with $k_{z}=1.25$ (see text).

Combined, the model equations constitute an eigenvalue problem describing the perturbed potential $\phi_{1}(z)$ in an inhomogeneous, magnetized, two-dimensional plasma undergoing shear flow. This problem was solved computationally for equilibrium velocity, collision frequency, and density profiles specified by:

$$
\begin{aligned}
v_{\circ}(z) & =V_{\circ} \operatorname{Tanh}(z / L) \\
v_{\text {in }}(z) & =v_{\circ}-v_{1} \operatorname{Tanh}(z / L) \\
n_{\circ}(z) & =N_{\circ} u \frac{v_{\circ} / v_{\text {in }}(z)}{u-v_{\circ}(z)}
\end{aligned}
$$

and shown to yield rapidly growing solutions even in the collisional regime $\left(\nu_{\text {in }} \gg|\omega|\right)$. Those results are reproduced here in Fig. 4, which shows how the instability growth rate increases with increments in $v_{1}$ and the increasingly steep number density profile that accompanies them.

An approximate, closed-form expression for the growth rate highlighting its dependence on background ionospheric parameters is derived below. In our analysis, we focus on the collisional branch of the instability thought to operate in the bottomside equatorial $F$ region by applying the limit $v_{\text {in }} \rightarrow$ $\infty$ in Eqs. (6) and (7), leading to:

$$
\begin{aligned}
& \frac{d}{d z}\left(v_{\text {in }} n_{\circ} \frac{d \phi_{1}}{d z}\right)-v_{\text {in }} n_{\circ} k_{x}^{2} \phi_{1} \\
& =-k_{x} \frac{d}{d z}\left(v_{\text {in }} \frac{u-v_{\circ}}{\omega-k_{x} v_{\circ}} \frac{d n_{\circ}}{d z} \phi_{1}\right)
\end{aligned}
$$

We next multiply the equation by $\phi_{1}^{*}$ and integrate over altitudes where the perturbed potential exists:

$$
\begin{aligned}
& -\int d z v_{\text {in }} n_{\circ}\left(\left|\phi_{1}^{\prime}\right|^{2}+k_{x}^{2}\left|\phi_{1}\right|^{2}\right) \\
& =k_{x} \int d z v_{\text {in }} \frac{u-v_{\circ}}{\omega-k_{x} v_{\circ}} n_{\circ}{ }^{\prime} \phi_{1} \phi_{1}^{* \prime}
\end{aligned}
$$

where the prime notation refers to differentiation with respect to $z$ and where integration by parts has been employed. Since the left side of (8) is real, the frequency $\omega=\omega_{r}+i \gamma$ can only be complex valued if the eigenfunction $\phi_{1}$ is also somewhere complex. This is a necessary condition for wave growth and affirms the conjecture of Hysell and Kudeki (2004) that the asymmetric flow pattern arising from the complex eigenfunction is a crucial feature of the collisional shear instability. Complex eigenfunctions guarantee that the wavefronts (surfaces of constant phase) that form and the convection that follows them are neither strictly vertical nor horizontal, either one of these geometries tending to be stable.

The real and imaginary parts of Eq. (8) yield integral equations for the wave frequency and growth rate that depend on the shape of the eigenfunction that are no easier to evaluate than was the original eigenvalue problem. The instability in question is inherently nonlocal and is sensitive to the ionospheric velocity, number density, and collision frequency profiles. However, the numerical calculations of $\mathrm{Hy}-$ sell and Kudeki (2004) suggested that in the vicinity of the shear node, where $v_{\circ}^{\prime \prime}$ vanishes and about which $\phi$ is localized, the complex potential can be approximated by $\phi(z) \approx$ $\phi_{\circ} \exp \left(i k_{z} z\right)$. Utilizing this approximation and neglecting $\omega_{r}-k_{x} v_{\circ}$ by comparison to $\gamma$ in this region, an expression for the growth rate $\gamma$ can be derived from the real part of Eq. (8):

$$
\begin{aligned}
\gamma & \approx \frac{k_{x} k_{z} \int d z v_{\text {in }}\left(u-v_{\circ}\right) n_{\circ}{ }^{\prime}\left|\phi_{1}\right|^{2}}{k^{2} \int d z v_{\text {in }} n_{\circ}\left|\phi_{1}\right|^{2}} \\
& =\frac{k_{x} k_{z}\left\langle v_{\text {in }}\left(u-v_{\circ}\right) n_{\circ}{ }^{\prime}\right\rangle}{k^{2}\left\langle v_{\text {in }} n_{\circ}\right\rangle}
\end{aligned}
$$

where the averaging is weighted by the norm of the potential, a function that generally peaks about one scale height below the shear node and extends for several scale heights above and below it. Note that the choice of $k_{x}$ affects the mode shape and the associated value of $k_{z}$ so that the dependence of the growth rate on the horizontal wavenumber is not apparent from this analysis.

Given mode shapes and values for $k_{z}$ derived from a complete boundary value analysis, Eq. (9) gives growth rate estimates in good agreement with the eigenvalues of the full model expressed by (6) and Eq. (7) (see points plotted in Fig. 4). Furthermore, Eq. (9) accounts for the results of a numerical shear instability simulation reported on by Hysell and Kudeki (2004), which produced growing waves with relatively long e-folding times $(\sim 50 \mathrm{~min}$.) despite the presence of a steep vertical density gradient and widely differing plasma and neutral drift speeds in the simulation. In that two-dimensional simulation, shear flow was induced with the introduction of an ad hoc constant conductivity term meant to represent electrical loading by an off-equatorial $E$ region plasma. The effective $E$ region conductivity shorted out the $F$ region dynamo in the valley region but not in the vicinity of the $F$ peak, the net result being vertical shear. The add hoc term would contribute significantly to the denominator of (9), which represents the factors that dissipate polarization charge, but not to the numerator, which represents factors that accumulate polarization charge. The $E$ region 
loading both caused essential relative plasma-neutral drifts and shorted out the polarization electric fields driven by the instability.

In view of the evident impact of off-equatorial effects on ionospheric stability, we have generalized (9), rewriting it in terms of a dipole magnetic coordinate system and flux-tube integrated variables:

$$
\gamma \approx \frac{\kappa_{\phi} \kappa_{p}\left\langle\frac{h_{\phi} h_{q}}{h_{p}} v_{\text {in }}\left(u-v_{\circ}\right) n_{\circ}{ }^{\prime}\right\rangle}{\kappa_{\phi}^{2}\left\langle\frac{h_{p} h_{q}}{h_{\phi}} v_{\text {in }} n_{\circ}\right\rangle+\kappa_{p}^{2}\left\langle\frac{h_{\phi} h_{q}}{h_{p}} v_{\text {in }} n_{\circ}\right\rangle}
$$

where the angle brackets now represent averages over a magnetic flux tube as well as over a range of apex altitudes, the prime represents a derivative with respect to the $p$ coordinate, and where the $\kappa$ terms are constant, nondimensional wavenumbers related to the wavenumber through the appropriate scale factor, e.g. $k_{\phi}=\kappa_{\phi} / h_{\phi}$.

In the absence of information regarding the mode shape of the perturbed potential, we postulate that one may still use Eqs. (9) or (10) to assess the stability of the ionosphere, assuming that the two wavenumber components are comparable and replacing the weighted average with a straight average over the bottomside $F$ region.

\section{Discussion}

New experimental capabilities at Jicamarca have focused attention on shear flow in the postsunset bottomside $F$ region ionosphere and the possible role it plays in the variability of equatorial spread $F$. While the existence of shear flow has been recognized for more than two decades, combined coherent and incoherent scatter observations presently allow us to locate the shear node and to assess the shear strength quantitatively. Shear flow exists for a period of several hours surrounding sunset and is most intense after sunset at low bottomside altitudes approaching the valley region. The scale length for the shear is measured in tens of kilometers there.

Shear flow arises mainly from the competition between the $E$ and $F$ region dynamos and from demands on the vertical boundary currents in the bottomside imposed from above by the imperfectly efficient $F$ region dynamo and from below by interruptions in the equatorial electrojet around the evening terminator (Haerendel et al., 1992; Haerendel and Eccles, 1992). Assessing the relative importance of these factors quantitatively is impeded by a lack of neutral wind profile measurements in the equatorial thermosphere as well as of off-equatorial $E$ and valley region conductivities.

One consequence of vertical shear is the existence of regions where the zonal plasma flow is strongly retrograde, i.e. in the direction opposite the local neutral wind. When horizontal conductivity gradients are also present in such a region, wind-driven interchange instabilities and plasma irregularities results. This now appears to be the explanation for bottom-type scattering layers, which occupy retrograde strata and appear when the tilted postsunset $F$ region supplies the necessary zonal conductivity gradient (Kudeki and
Bhattacharyya, 1999). It should be noted that the existence of vigorous wind-driven interchange instabilities in the bottomside does not by itself imply that $E$ region conductivity loading on the associated magnetic flux tubes is insignificant. Hysell et al. (2004) recently showed that the relatively small wavelengths of the bottom-type plasma irregularities, combined with finite parallel conductivity, would allow the irregularities to grow even on flux tubes where the $E$ region dynamo is dominant.

On occasions when topside spread $F$ occurs, the bottomtype layers seem to be organized in clusters separated by about $30 \mathrm{~km}$. We interpret this modulation as evidence of decakilometric undulations or waves in the bottomside $F$ region. The wind-driven instabilities would be stable and unstable in the different phases of such waves. In several instances, the topside irregularities that followed also exhibited structuring with this characteristic scale size. The decakilometric waves therefore appear to serve as seed waves for full-blown spread $F$. The existence of seed waves is often postulated to explain the rapid appearance and development of radar plumes and intense plasma irregularities shortly after sunset in the equatorial ionosphere. Gravity waves propagating into the thermosphere may seed the ionosphere as well but are not expected to preferentially excite waves at the observed wavelength. The $\sim 30 \mathrm{~km}$ wavelengths is itself unrelated to interchange instability, posing further questions about its origin. That the shear scale length is also measured in tens of kilometers suggests a connection.

Beyond creating conditions for wind-driven instabilities, shear flow may itself affect the stability of the bottomside $F$ region. Boundary value analyses by Guzdar et al. (1983), Huba and Lee (1983), and Satyanarayana et al. (1984) indicated that shear flow generally stabilizes ionospheric interchange instabilities. However, Fu et al. (1986), Ronchi et al. (1989), and Flaherty et al. (1999) challenged this idea by pointing out the limitations of boundary value analysis, which is incomplete when applied to non-normal models like those describing sheared flows. The issue of shear flow stabilization remains contentious, and a number of recent theoretical and computational studies continue to support the idea (Hassam, 1992; Sekar and Kelley, 1998; Chakrabarti and Lakhina, 2003). Chakrabarti and Lakhina (2001) further showed that the nonlinear evolution of the Rayleigh-Taylor instability itself induces shear flow which contributes to the saturation of the instability.

However, by extending the work of Keskinen et al. (1988) to the equatorial zone, Hysell and Kudeki (2004) showed that a collisional shear instability may exist in the postsunset ionosphere and be responsible for the precursor waves. In the collisional limit, retrograde plasma motion rather than shear per se drives the instability. However, it is not just a variant of the standard wind-driven interchange instability, since instability occurs when the neutral wind and the background density gradient are normal to one another. The instability is the collisional branch of electrostatic Kelvin Helmholtz and is therefore aptly termed a collisional shear instability. The instability is not damped by collisions and does not require 
a prereversal enhancement and the elevation of the $F$ layer to function. This may allow it to operate prior to the onset of gravitational interchange instabilities, which are stabilized by collisions, and thereby to serve as a source of seed waves.

An approximate linear growth rate expression for the collisional shear instability has been derived. The inherently nonlocal nature of the instability prevents the derivation of an exact expression, but the approximation reveals at least how the growth rate scales with background parameters. Retrograde plasma motion and a steep vertical plasma density gradient in the retrograde stratum are conducive to wave growth. Large flux-tube integrated plasma conductivity suppresses growth. Whether the former overcomes the latter depends on the strength of the neutral wind, the cause of retrograde drifts, and the off-equatorial conductivity distribution.

For example, if the boundary current condition is mainly responsible for retrograde drifts in the bottomside, then the bottomside flux tubes need not be heavily loaded by conductivity in the off-equatorial $E$ and valley regions, and shear instability could be robust. If shear flow is mainly induced by a strong $E$ region dynamo controlling flux tubes in the bottomside, however, then the shear instability would likely be damped. The existence of irregularities in the bottom-type layers gives no clue about which scenario is operating, since they can exist in either case.

Thermospheric wind measurements made by the C/NOFS satellite will be critical for quantifying the shear instability theory. In order to evaluate (10), information about the off-equatorial conductivity distribution will also be required. This could be provided by a meridional chain of sounders, a bistatic HF sounder link, or by satellite occultation experiments. The same information is required both to quantify the causes of shear flow in the equatorial ionosphere and to evaluate the growth rate of the gravitational interchange instabilities responsible for spread $F$.

Acknowledgements. This work was supported by the National Science Foundation through cooperative agreement ATM-0432565 to Cornell University and by NSF grant ATM-0225686 to Cornell University. Additional support was received from the Air Force Research Laboratory through award 03C0067. The Jicamarca Radio Observatory is operated by the Instituto Geofísico del Perú, Ministry of Education, with support from the NSF cooperative agreements just mentioned. The help of the staff was much appreciated.

Topical Editor M. Pinnock thanks two referees for their help in evaluating this paper.

\section{References}

Anandarao, B. G., Raghavararao, R., Desai, J. N., and Haerendel, G.: Vertical winds and turbulence over Thumba, J. Atmos. Terr. Phys., 40, 157-163, 1978.

Chakrabarti, N. and Lakhina, G. S.: Nonlinear saturation of Rayleigh-Taylor instability and generation of shear flow in equatorial spread-F plasma, Nonlin. Proc. in Geophys., 8, 181-190, 2001.

Chakrabarti, N. and Lakhina, G. S.: Collisional Rayleigh-Taylor instability and shear-flow in equatorial spread $F$ plasma, Ann.
Geophys., 21, 1153-1157, 2003,

SRef-ID: 1432-0576/ag/2003-21-1153.

Eccles, J. V.: A simple model of low-latitude electric fields, J. Geophys. Res., 103, 26 699-26 708, 1998.

Eccles, J. V., Maynard, N., and Wilson, G.: Study of the evening drift vortex in the low-latitude ionosphere using San Marco electric field measurements, J. Geophys. Res., 104, 28 133-28 143, 1999.

Farley, D. T., Bonelli, E., Fejer, B. G., and Larsen, M. F.: The prereversal enhancement of the zonal electric field in the equatorial ionosphere, J. Geophys. Res., 91, 13 723-13 728, 1986.

Fejer, B. G.: The equatorial ionospheric electric fields. A review, J. Atmos. Terr. Phys., 43, 377-386, 1981.

Flaherty, J. P., Seyler, C. E., and Trefethen, L. N.: Large-amplitude transient growth in the linear evolution of equatorial spread $F$ with a sheared zonal flow, J. Geophys. Res., 104, 6843-6858, 1999.

Fu, Z. F., Lee, L. C., and Huba, J. D.: A quasi-local theory of the $\mathbf{E} \times \mathbf{B}$ instability in the ionosphere, J. Geophys. Res., 91, 32633269, 1986.

Guzdar, P. N., Satyanarayana, P., Huba, J. D., and Ossakow, S. L.: Influence of velocity shear on Rayleigh-Taylor instability, Geophys. Res. Lett., 9, 547-550, 1983.

Haerendel, G. and Eccles, J. V.: The role of the equatorial electrojet in the evening ionosphere, J. Geophys. Res., 97, 1181-1192, 1992.

Haerendel, G., Eccles, J. V., and Cakir, S.: Theory for modeling the equatorial evening ionosphere and the origin of the shear in the horizontal plasma flow, J. Geophys. Res., 97, 1209-1223, 1992.

Hassam, A. B.: Nonlinear stabilization of the Rayleigh-Taylor instability by external velocity shear, Phys. Fluids, 4, 485-487, 1992.

Heelis, R. A., Kendall, P. C., Moffet, R. J., Windle, D., and Rishbeth, H.: Electric coupling of the $E$ and $F$ regions and its effect of the $F$ region drifts and winds, Planet. Space Sci., 22, 743-756, 1974.

Huba, J. D. and Lee, L. C.: Short wavelength stabilization of the gradient drift instability due to velocity shear, Geophys. Res. Lett., 10, 357-360, 1983.

Hysell, D. L. and Kudeki, E.: Collisional shear instability in the eqautorial $F$ region ionosphere, J. Geophys. Res., 109(A11 301), 2004.

Hysell, D. L., Chun, J., and Chau, J. L.: Bottom-type scattering layers and equatorial spread F, Ann. Geophys., 22, 4061-4069, 2004, SRef-ID: 1432-0576/ag/2004-22-4061.

Kelley, M. C., Larsen, M. F., and La Hoz, C.: Gravity wave interaction of equatorial spread $F$ : A case study, J. Geophys. Res., 86, 9087-9100, 1981.

Keskinen, M. J., Mitchell, H. G., Fedder, J. A., Satyanarayana, P., Zalesak, S. T., and Huba, J. D.: Nonlinear evolution of the Kelvin-Helmholtz instability in the high-latitude ionosphere, J. Geophys. Res., 93, 137-152, 1988.

Kudeki, E. and Bhattacharyya, S.: Post-sunset vortex in equatorial $F$-region plasma drifts and implications for bottomside spread$F$, J. Geophys. Res., 104, 28 163-28 170, 1999.

Kudeki, E. and Sürücü, F.: Radar interferometric imaging of fieldaligned plasma irregularities in the equatorial electrojet, Geophys. Res. Lett., 18, 41-44, 1991.

Kudeki, E., Fejer, B. G., Farley, D. T., and Ierkic, H. M.: Interferometer studies of equatorial $F$ region irregularities and drifts, Geophys. Res. Lett., 8, 377-380, 1981. 
Kudeki, E., Bhattacharyya, S., and Woodman, R. F.: A new approach in incoherent scatter $F$ region E x B drift measurements at Jicamarca, J. Geophys. Res., 104, 28 145-28 162, 1999.

Larsen, M. F. and Odom, C. D.: Observations of altitudinal and latitudinal $E$-region neutral wind gradients near sunset at the magnetic equator, Geophys. Res. Lett., 24, 1711-1714, 1997.

Maruyama, T., Nozaki, K., Yamamoto, M., and Fukao, S.: Ionospheric height changes at two closely separated equatorial stations and implications in spread $F$ onsets, J. Atmos. Sol. Terr. Phys., 64, 1557-1563, 2002.

Richmond, A. D.: Equatorial electrojet, I, Development of a model including winds and instabilities, J. Atmos. Terr. Phys., 35, 1083-1103, 1973.

Ronchi, C., Similon, P. L., and Sudan, R. N.: A nonlocal linear theory of the gradient drift instability in the equatorial electrojet, J. Geophys. Res., 94, 1317-1326, 1989.

Satyanarayana, P., Guzdar, P. N., Huba, J. D., and Ossakow, S. L.: Rayleigh-Taylor instability in the presence of a stratified shear layer, J. Geophys. Res., 89, 2945-2954, 1984.
Sekar, R. and Kelley, M. C.: On the combined effects of vertical shear and zonal electric field patterns on nonlinear equatorial spread $F$ evolution, J. Geophys. Res., 103, 20,735-20,748, 1998.

Stenning, R. J.: A two-layer ionospheric dynamo circulation, J. Geophys. Res., 86, 3543-3550, 1981.

Takeda, M. and Maeda, H.: $F$ region dynamo in the evening Interpretation of equatorial $\Delta \mathrm{D}$ anomaly found by MAGSAT, J. Atmos. Sol. Terr. Phys., 45, 401-408, 1983.

Tsunoda, R. T., Livingston, R. C., and Rino, C. L.: Evidence of a velocity shear in bulk plasma motion associated with the postsunset rise of the equatorial $F$ layer, Geophys. Res. Lett., 8, 807810, 1981.

Vadas, S. L. and Fritts, D. C.: Thermospheric response to gravity waves arising from mesoscale convective complexes, J. Atmos. Sol. Terr. Phys., 66, 781-804, 2004.

Woodman, R. F.: Vertical drift velocities and east-west electric fields at the magnetic equator, J. Geophys. Res., 75, 6249-6259, 1970.

Woodman, R. F.: Coherent radar imaging: Signal processing and statistical properties, Radio Sci., 32, 2373-2392, 1997. 